\title{
An Essential Role for Histone Deacetylase 4 in Synaptic Plasticity and Memory Formation
}

\author{
Mi-Sung Kim, ${ }^{1 \star}$ M. Waseem Akhtar, ${ }^{2 \star}$ Megumi Adachi, ${ }^{2}$ Melissa Mahgoub,${ }^{2}$ Rhonda Bassel-Duby, ${ }^{1}$ Ege T. Kavalali, ${ }^{3}$ \\ Eric N. Olson, ${ }^{1}$ and Lisa M. Monteggia ${ }^{2}$ \\ Departments of ${ }^{1}$ Molecular Biology, ${ }^{2}$ Psychiatry, and ${ }^{3}$ Neuroscience, University of Texas Southwestern Medical Center, Dallas, Texas 75390
}

Histone deacetylases (HDACs), a family of enzymes involved in epigenetic regulation, have been implicated in the control of synaptic plasticity, as well as learning and memory. Previous work has demonstrated administration of pharmacological HDAC inhibitors, primarily those targeted to class I HDACs, enhance learning and memory as well as long-term potentiation. However, a detailed understanding of the role of class II HDACs in these processes remains elusive. Here, we show that selective loss of Hdac4 in brain results in impairments in hippocampal-dependent learning and memory and long-term synaptic plasticity. In contrast, loss of Hdac5 does not impact learning and memory demonstrating unique roles in brain for individual class II HDACs. These findings suggest that HDAC4 is a crucial positive regulator of learning and memory, both behaviorally and at the cellular level, and that inhibition of Hdac4 activity may have unexpected detrimental effects to these processes.

\section{Introduction}

Epigenetic mechanisms control accessibility of chromatin to transcriptional factors in neurons, which in turn impact gene expression and ultimately regulate neurotransmission, synaptic plasticity, as well as disease states (Abel and Zukin, 2008; Borrelli et al., 2008; Jiang et al., 2008). A key epigenetic modification involves the posttranslational removal or addition of an acetyl group to specific lysine residues in the $\mathrm{N}$ terminus of histone residues, which is accomplished by histone deacetylases (HDACs) and histone acetytransferases (HATs), respectively. Alterations in histone acetylation in animal models has been implicated in learning and memory as well as synaptic plasticity (Alarcón et al., 2004; Korzus et al., 2004; Levenson et al., 2004; Vecsey et al., 2007), with HDAC inhibitors facilitating these processes in wild-type mice and mouse models of neurodegenerative diseases (Fischer et al., 2007). Studies based on pharmacological HDAC inhibitors have primarily focused on class I HDAC family members (HDAC1, HDAC2, HDAC3, and HDAC8), with recent data suggesting that brain deletion of $\mathrm{Hdac} 2$ facilitates memory formation and synaptic plasticity (Guan et al., 2009). However, the role of class IIa HDACs (HDAC4, HDAC5, HDAC7, and HDAC9), which act as transcriptional repressors and shuttle between the nucleus and cytoplasm in response to intracellular sig-

\footnotetext{
Received May 1, 2012; revised May 3, 2012; accepted May 30, 2012.

Author contributions: R.B.-D., E.T.K., E.N.O., and L.M.M. designed research; M.-S.K., M.W.A., M.A., and M.M. performed research; M.-S.K., M.W.A., M.A., and M.M. analyzed data; M.-S.K., M.W.A., and L.M.M. wrote the paper. This work was supported by National Institutes of Health Grants MH081060 (L.M.M.) and MH066198 (E.T.K.) and The Robert A. Welch Foundation Grant I-0025 (E.N.O.).

The authors declare no competing financial interests.

*M.-S.K. and M.W.A. contributed equally to this work

Correspondence should be addressed to Lisa M. Monteggia, Department of Psychiatry, University of Texas Southwestern Medical Center, 5323 Harry Hines Boulevard, Dallas, TX 75390-9070. E-mail: lisa.monteggia@utsouthwestern.edu.

DOI:10.1523/JNEUROSCI.2089-12.2012

Copyright $\odot 2012$ the authors $\quad 0270-6474 / 12 / 3210879-08 \$ 15.00 / 0$
}

naling (Haberland et al., 2009), has not been addressed in these processes.

HDAC4 and HDAC5 are highly enriched in the brain with strong expression in broad forebrain regions such as hippocampus, cortex, and amygdala (Broide et al., 2007), suggesting they may be important in underlying enhanced learning and memory as well as synaptic plasticity observed with broad HDAC inhibitors. Constitutive $H d a c 5^{-1-}$ knock-out (KO) mice show enhanced reward to cocaine in conditioned place preference (Renthal et al., 2007). Conversely, overexpression of Hdac4 or Hdac5 attenuates the rewarding effects of cocaine in conditioned place preference (Kumar et al., 2005; Renthal et al., 2007). Collectively, these data have implicated HDAC4 and HDAC5 as negative regulators of rewarding related behavior. The maintenance of reward-related behavior likely involves alterations in synaptic plasticity, although specific roles for HDAC4 and HDAC5 in these specific processes, as well as in learning and memory related tasks remain unexplored.

The goal of this study was to investigate the potential involvement of HDAC4 and HDAC5 in learning, memory, and synaptic plasticity. Constitutive $H d a c 5^{-1-}$ null mice are viable, while in contrast constitutive Hdac4 mice die perinatally (Vega et al., 2004). We therefore generated mice with conditional brainspecific Hdac4 deletion and show that these animals have alterations in motor coordination and anxiety, as well as significant deficits in learning tasks. The learning and memory deficits in the brain-specific Hdac4 knock-out mice were associated with significant impairments in long-term potentiation (LTP) induction in the hippocampus without alterations in basal synaptic transmission. In contrast, $\mathrm{Hdac} 5^{-1-}$ mice display no abnormalities in these behaviors, including learning and memory. These findings establish a surprising and key role of HDAC4, but not HDAC5, as a positive regulator of memory formation and long-term synaptic plasticity. 


\section{Materials and Methods}

Generation of knock-out mice. Constitutive Hdac4 mice die perinatally (Vega et al., 2004), precluding the ability to examine them for behavioral deficits. We therefore generated conditional brain-specific Hdac4 knockout mice. Floxed Hdac4 mice $\left(H d a c 4^{\text {loxP/loxP }}\right)$ were generated by flanking exon 5 with loxP sites, which resulted in an out-of-frame mutation in the Hdac4 allele (Potthoff et al., 2007). To generate mice lacking Hdac4 in the brain, mice heterozygous for the Hdac4 ${ }^{\text {loxP }}$ allele were mated to CaMKIICre transgenic mice that express Cre recombinase specifically in the forebrain beginning at $\sim 10 \mathrm{~d}$ of age (Luikart et al., 2005). The resulting mice were crossed to homozygous Hdac4 floxed mice (Hdac4 $4^{\text {loxP/loxP }}$ ) to generate forebrain-specific conditional null mice (Hdac4 ${ }^{\text {loxP/loxP }}$; CaMKII$\mathrm{Cre}$ ). The conditional Hdac4 brain-specific knock-out (Hdac4bko) mice were genotyped by PCR. The primers for detection of Hdac4 are as follows: HDAC4, forward, 5'-ATC TGC CCA CCA GAG TAT GTG-3'; HDAC4, reverse, 5' -CTT GTT GAG AAC AAA CTC CTG CAG CT-3'; and nLacZ, reverse, $5^{\prime}$-GAT TGA CCG TAA TGG GAT AGG TTA CG$3^{\prime}$. The primers for Cre recombinase are as follows: $5^{\prime}$-AGG TTC GTT CAC TCA TGG A- $3^{\prime}$ and $5^{\prime}$-TCG ACC AGT TTA GTT ACC C- $3^{\prime}$. The constitutive $H d a c 5^{-1-}$ mice had been previously generated and the deletion confirmed (Chang et al., 2004). The Hdac5 $5^{-1-}$ and wt littermate controls were generated by heterozygous $\times$ heterozygous crosses.

Histology. Mice were transcardially perfused, and the brains were removed and fixed overnight in DEPC-treated $4 \%$ paraformaldehyde. The brains were paraffin-embedded, sectioned at $5 \mu \mathrm{m}$, and mounted on gelatin-coated slides. For in situ hybridization, riboprobes were labeled with $\left[\alpha-{ }^{35} \mathrm{~S}\right] \mathrm{UTP}$ using the MAXIscript in vitro transcription kit (Ambion) following the manufacturer's instructions. In situ hybridization of sectioned tissues was performed as previously described (Shelton et al., 2000). The Hdac4 riboprobe was originally generated by PCR using the following primers: forward, 5' -CAG AAG CTG CAG CAG CTC AAG-3', and reverse, 5'-GGT GGA GAG CTC TGG TCA AGG-3'.

$R N A$ isolation and quantitative RT-PCR. RNA was isolated from cortex, hippocampus, and cerebellum using the RNeasy kit (QIAGEN) according to the manufacturer's instructions. Two micrograms of RNA were converted to cDNA using random primers and SuperScript III Reverse Transcriptase (Invitrogen). Quantitative analysis was performed by real-time PCR using the ABI PRISM 7000 sequence detection system with TaqMan primers (Applied Biosystems) or with SYBR Green Master Mix reagent (Applied Biosystems). To quantify the relative changes in gene expression, the comparative Ct method was used (Livak and Schmittgen, 2001). A fold change was calculated by the following equation: $2^{-\Delta \Delta \mathrm{Ct}}=\left[\left({ }^{\Delta \Delta} \mathrm{Ct} H d a c-\mathrm{Ct} \text { Gapdh }\right)_{\text {knock-out }}\right.$ - (Ct Hdac - Ct Gapdh $\left.)_{\text {wild type }}\right]$.

Behavioral overview. All behavior testing was done on male mice at least 2 months of age that were littermates. The mice were on a $12 \mathrm{~h}$ light/dark cycle with ad libitum access to food and water. Mice were allowed to habituate in the behavior room for $1 \mathrm{~h}$ before all behavioral testing. All data were analyzed and scored by an observer blind to the genotype. For all experiments, data were presented as mean \pm SEM, and Student's $t$ test was used to analyze data, unless otherwise noted, with significance set as $p<0.05$. All animal experimental procedures were reviewed and approved by the Institutional Animal Care and Research Advisory Committee at University of Texas Southwestern Medical Center.

Locomotor activity. Mice were placed in a fresh home cage and locomotor activity was measured for $2 \mathrm{~h}$ using four photocell beams linked to computer data acquisition software (San Diego Instruments). Ambulation was scored as horizontal movement by the animal within the $2 \mathrm{~h}$ of testing. Data were analyzed with repeated ANOVA.

Rotarod. Each mouse was placed on the activated rotarod (IITC Life Science), and its speed ramped up from 0 to $45 \mathrm{rpm}$ in $60 \mathrm{~s}$. The time for the mouse to fall off the rotarod or turn one full revolution was measured for four consecutive trials. After the test, the mouse was returned to its original cage for $\sim 24 \mathrm{~h}$. The test was repeated for a total of four runs the next day. Data were analyzed with repeated ANOVA.

Elevated plus maze. Mice were placed in the center of an elevated plus maze under dim lighting, and their behavior was monitored for $5 \mathrm{~min}$ using a video-tracking system (Ethovision 3.0). The time spent in the center, closed and open arms was determined.

Open field. Mice were videotaped under dim lighting for their activity during $5 \mathrm{~min}$ in a $42 \mathrm{~cm}$ square open field. The time spent in the center, the broader area of the center, and borders of the open field (periphery) was determined.

Fear conditioning. The fear conditioning paradigm was performed as described previously (Monteggia et al., 2004; Barbosa et al., 2008). Briefly, mice were placed in individual chambers (MED Associates) for 12 min during which time they received four scrambled footshocks at 0.5 $\mathrm{mA}$ for $2 \mathrm{~s}$. The footshocks were administered at 2.5, 5, 9, and $11.5 \mathrm{~min}$ after placement of the animals into the chamber. After the 12 min training session, the mice were immediately removed and placed back into their home cages. Ninety minutes later, short-term memory (STM) formation was tested. The mice were placed back in the same chamber for 3 min (no shock), and their freezing behavior was assessed by an observer blind to the genotypes. To examine long-term memory (LTM) formation, the mice were placed back in the same chamber $24 \mathrm{~h}$ after the training, and their freezing behavior was assessed for $3 \mathrm{~min}$. The freezing behavior was defined as no movement except for respiration. To assess pain sensitivity to the footshock, mice were placed back in the individual chambers for $2 \mathrm{~min}$ to habituate. The animals were then shocked $(0.05$ $\mathrm{mA}$ for $1 \mathrm{~s}$ ), and their behavior was scored as no movement, flinching, or jumping. Every $30 \mathrm{~s}$, the shock was increased by $0.05 \mathrm{~mA}$, with a maximum shock of $0.6 \mathrm{~mA}$, until the animal flinched and jumped in response to the shock.

Morris water maze. A circular pool was filled with room temperature water to a depth of $\sim 12$ inches. A platform ( $10 \mathrm{~cm}$ diameter) was placed in one quadrant of the pool with the top of the platform $\sim 2 \mathrm{~cm}$ below the water level. White nontoxic paint was added to enhance the contrast with the animal and to hide the location of the platform. Each day, the mice were placed in the pool and allowed to swim for $1 \mathrm{~min}$ to find the platform. The swim path, swim velocity, and time spent in each quadrant were obtained using automated video-tracking software from Noldus (Ethovision 2.3.19). If the mouse did not find the platform within a minute, they were gently guided or placed on the platform for $10 \mathrm{~s}$, and then removed from the pool and returned to their home cage. Each animal was placed in the pool for a total of four times, starting from a different quadrant for each trial, each day for $10 \mathrm{~d}$. Immediately following the training days, a probe test was conducted in which the platform is removed from the pool and each mouse was allowed to swim for $1 \mathrm{~min}$ to determine whether the animal had learned the location of the platform.

Slice electrophysiology. For extracellular field recordings, 2- to 5 -month-old mice were used. Mice were anesthetized by intraperitoneal Nembutal injection and decapitated. The brain was rapidly dissected, and hippocampal slices $(400 \mu \mathrm{m})$ were collected in ice-cold dissection buffer containing the following (in $\mathrm{mM}$ ): 212 sucrose, $3 \mathrm{KCl}, 5 \mathrm{MgCl}_{2}, 0.5$ $\mathrm{CaCl}_{2}, 1 \mathrm{NaH}_{2} \mathrm{PO}_{4}, 26 \mathrm{NaHCO}_{3}$, and 10 glucose. CA3 region was cut to avoid epileptiform activity. Slices were placed at $30^{\circ} \mathrm{C}$ for $2 \mathrm{~h}$ in artificial CSF (ACSF) containing the following (in $\mathrm{mm}$ ): $124 \mathrm{NaCl}, 5 \mathrm{KCl}, 26$ $\mathrm{NaHCO}_{3}, 1.25 \mathrm{NaH}_{2} \mathrm{PO}_{4}, 2 \mathrm{CaCl}_{2}, 1 \mathrm{MgCl}_{2}$, and 10 glucose. ACSF and dissection buffer were bubbled with $95 \% \mathrm{O}_{2} / 5 \% \mathrm{CO}_{2}$. Before recordings, slices were placed in a submersion-recording chamber, maintained at $30^{\circ} \mathrm{C}$, and perfused with ACSF. Concentric, bipolar tungsten electrodes were used to activate Schaffer collateral/commissural (SC) fibers in the hippocampal CA1 region. Extracellular glass microelectrodes filled with ACSF (resistance, $\sim 1 \mathrm{M} \Omega$ ) were placed in the stratum radiatum to measure field fEPSPs. For baseline recordings, slices were stimulated at 0.033 $\mathrm{Hz}$ for $20 \mathrm{~min}$ at stimulation intensities of $40-50 \%$ of the highest measured fEPSP size. LTP was induced by applying a theta burst stimulus consisting of three bursts (10 s interval), each composed of 15 trains (5 $\mathrm{Hz}$ ) with four pulses $(100 \mathrm{~Hz})$. Paired-pulse facilitation (PPF) was tested by applying two pulses with interstimulus intervals ranging from 20 to 200 ms. An Axoclamp 2B amplifier (Molecular Devices) was used for experiments. Data were sampled at $5 \mathrm{kHz}$ and analyzed using a program written in LabView (National Instruments). For experiments, data were presented as mean \pm SEM.

Cell culture. Primary hippocampal neurons were cultured as previously described (Kavalali et al., 1999). Briefly, whole hippocampi were 
A

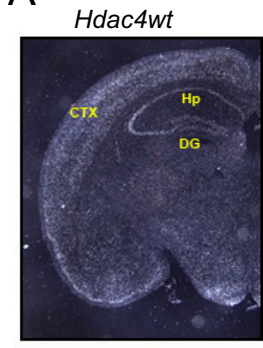

C



B

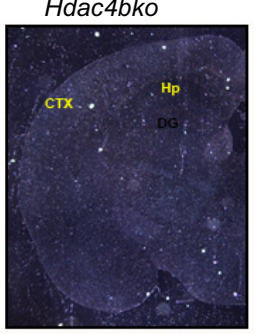

D

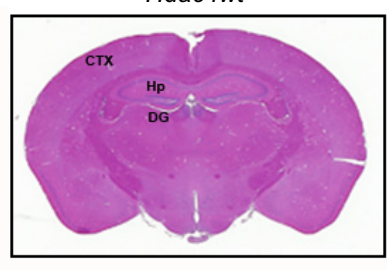

Hippocampus Cortex Cerebellum wt bko bko wt bko bko wt bko bko
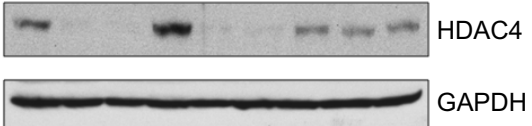

Figure 1. Generation of forebrain-specific Hdac4bko mice. A, Detection of $H d a c 4$ transcripts by in situ hybridization in coronal sections from Hdac $4 w t$ and $H d a c 4 b k o$ mice at 4 months of age. $\boldsymbol{B}$, Western blots of protein from brain lysates showed deletion of HDAC4 in the cortex and hippocampus, but not the cerebellum of Hdac4bko mice. GAPDH protein was used as a loading control. $C$, Expression levels of class II HDAC mRNAs (Hdac4, Hdac5, Hdac7, and Hdac9) in the hippocampus of Hdac4bko mice compared with Hdac $4 w t$, as determined by quantitative RT-PCR. Error bars indicate \pm SEM. ${ }^{*} p<0.05$. D, Hematoxylin and eosin staining showed no obvious changes in brain morphology between 4-month-old Hdac4bko and Hdac4wt mice. CTX, Cortex; DG, dentate gyrus; Hp, hippocampus.

isolated from homozygous $\mathrm{Hdac} 4^{\text {loxP/loxP }}$, or constitutive $H d a c 5^{-1-} \mathrm{KO}$ mice on postnatal days $0-1$. After dissection, tissues were trypsinized for $10 \mathrm{~min}$ at $37^{\circ} \mathrm{C}$, mechanically dissociated using siliconized glass pipettes, and plated onto Matrigel-coated coverslips. A concentration of $4 \mu \mathrm{M}$ cytosine arabinoside (Sigma-Aldrich) was added at $1 \mathrm{~d}$ in vitro (DIV) and reduced to a concentration of $2 \mu \mathrm{M}$ at $4 \mathrm{DIV}$.

Lentivirus production. Lentivirus was generated as previously described (Dittgen et al., 2004; Nelson et al., 2006; Akhtar et al., 2009). Briefly, HEK 293 cells were transfected with the expression plasmid, pFUGW or pFUGW-Cre, and two helper plasmids, $\Delta 8.9$ and vesicular stomatitis virus G-protein, at $3 \mu \mathrm{g}$ of each DNA per $75 \mathrm{~cm}^{2}$ flask using the Fugene 6 transfection system (Roche Molecular Biochemicals). Lentiviruscontaining culture medium was harvested $48 \mathrm{~h}$ after transfection, filtered at a $0.45 \mu \mathrm{m}$ pore size, and immediately used for infection. Hippocampal cultures were infected at 4 DIV by adding $300 \mu \mathrm{l}$ of viral suspension to each well containing $700 \mu \mathrm{l}$ of media, and recordings were done at 14-17 DIV.

In vitro electrophysiology recordings. Synaptic activity was recorded from hippocampal pyramidal neurons (in at least three independent cultures) using a whole-cell voltage-clamp technique. Data were acquired using an Axopatch 200B amplifier and Clampex 9.2 software (Molecular Devices). Recordings were filtered at $2 \mathrm{kHz}$ and sampled at $200 \mu$ s. A modified Tyrode's solution containing the following (in $\mathrm{mM}$ ): $150 \mathrm{NaCl}, 4 \mathrm{KCl}, 2 \mathrm{MgCl}_{2}, 2 \mathrm{CaCl}_{2}, 10$ glucose, and 10 HEPES, pH 7.4, was used as external bath solution, with $50 \mu \mathrm{M}$ picrotoxin and $1 \mu \mathrm{M}$ TTX to isolate miniature EPSCs (mEPSCs). The pipette internal solution for the voltage-clamp experiments contained the following (in $\mathrm{mM}$ ): $115 \mathrm{Cs}-\mathrm{MeSO}_{3}, 10 \mathrm{CsCl}, 5 \mathrm{NaCl}, 10$ HEPES, 0.6 EGTA, 20 TEA-Cl, 4 Mg-ATP, $0.3 \mathrm{Na}_{3} \mathrm{GTP}$, pH 7.35, and 10 QX-314 (300 mOsm). Statistical analysis was performed using Student's $t$ test. Statistical significance was defined as $p<0.05$. For experiments, data were presented as mean \pm SEM.

\section{Results}

\section{Generation of brain-specific $H$ dac 4 knock-out mice}

To generate mice lacking $\mathrm{Hdac} 4$ specifically in forebrain neurons, we mated mice carrying a homozygous floxed $H$ dac $4^{\text {loxP/loxP }}$ allele (Potthoff et al., 2007) with mice harboring a transgene that GAPDH

expresses Cre recombinase under the control of the calcium/calmodulin-dependent protein kinase II promoter (CaMKII-Cre). The CaMKII-Cre transgenic mouse expresses Cre recombinase from postnatal day 10 in forebrain regions, including the neocortex and the limbic system, but not in the cerebellum (Luikart et al., 2005). Mice with homozygous deletion of Hdac4 in the forebrain (hereafter referred to as $H d a c 4 b k o$ mice for $\mathrm{Hdac} 4$ brain $\mathrm{KO}$; wildtype littermate controls are represented as Hdac4wt mice) were born at expected Mendelian ratios and showed normal body weight, gross brain morphology, and life span (data not shown). Successful deletion of Hdac4 in the brain of mutant mice was validated by in situ hybridization, Western blot analysis, and quantitative RT-PCR. In Hdac4bko mice, Hdac4 mRNA expression (Fig. $1 A$ ) and protein levels (Fig. $1 B$ ) were dramatically reduced in the forebrain, including the cortex, amygdala, and hippocampus, but not in the cerebellum. To identify whether the loss of Hdac4 impacted other class II HDACs, we examined the expression of Hdac5, Hdac7, and Hdac9. We found that Hdac5, Hdac7, and Hdac9 mRNAs were not significantly altered in the hippocampus of Hdac $4 b k o$ compared with littermate Hdac4wt mice, suggesting that there were no significant compensatory changes in expression of these functionally related genes (Fig. 1C). We also examined the expression of class I HDACs in the hippocampus of Hdac4bko compared with littermate Hdac4wt mice, and no significant change in Hdac1 $(110 \pm 15.2 \%)$, Hdac2 $(124 \pm 16.8 \%)$, or Hdac3 $(118 \pm$ $15.1 \%$ ) mRNA was observed. Hematoxylin and eosin staining of brain sections did not reveal any detectable aberrations in brain morphology of the Hdac4bko compared with Hdac4wt mice (Fig. 1D).

\section{Hdac4bko mice display behavioral learning and memory impairments}

To determine whether the loss of Hdac4 in brain impacts behavior, the Hdac4bko mice were tested in several paradigms. We examined motor coordination using the rotarod test and found the Hdac $4 b k o$ mice exhibited a significant impairment relative to Hdac4wt mice (Fig. 2A). The deficits in motor coordination, with normal Hdac4 expression in the cerebellum (Fig. $1 B$ ), suggest that it is not loss of Hdac4 expression in the cerebellum that is contributing to the phenotype. In previous work, we demonstrated that this Cre driver line results in motor coordination deficits in the Rett syndrome Mecp2 mouse, without alterations in Mecp2 expression in the cerebellum, suggesting that altered gene loss independent of the cerebellum can impact this behavioral task (Gemelli et al., 2006).

Total locomotor activity was assessed for $120 \mathrm{~min}$, and the Hdac4bko mice were significantly hyperactive compared with Hdac4wt mice (Fig. $2 \mathrm{~B}$, inset). To better delineate the alteration in locomotor activity, we analyzed the data in 5 min increments and detected significant differences between Hdac4bko and Hdac4wt mice throughout this time period (Fig. $2 B$ ).

To assess whether the loss of Hdac4 impacts anxiety-related behavior, we tested mice in the elevated plus maze and the open- 

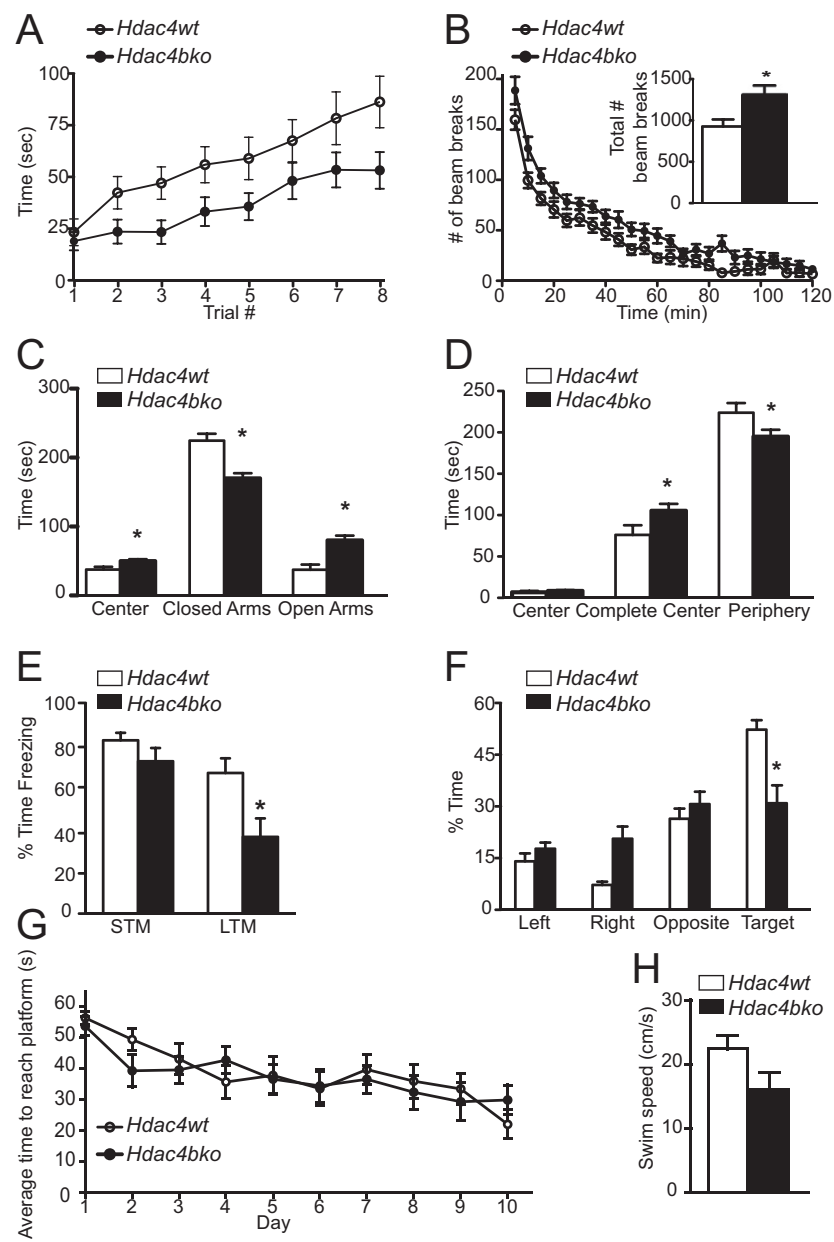

Figure 2. Hdac4bko mice show behavioral abnormalities including impaired memory formation. $\boldsymbol{A}$, The Hdac4bko mice were tested on the rotarod for motor coordination. Based on repeated measures with mixed-model analysis, there were significant group effects between Hdac4bko and Hdac4wt mice $\left(F_{(1,39)}=4.69\right.$; value of $\left.p=0.0366\right)$ and trial effect $\left(F_{(7,272)}=\right.$ 23.59; value of $p<0.0001)$. $\boldsymbol{B}, H d a c 4 b k 0$ mice display an increase in locomotor activity, as assessed by an increase in the number of beam breaks over $120 \mathrm{~min}$. The Hdac4bko had a significant increase in the total number of beam breaks over 120 min compared with Hdac4wt mice (inset). Total locomotor activity was assessed for $120 \mathrm{~min}$, and a significant total ambulation difference between the $\mathrm{Hdac} 4 b \mathrm{kO}$ and $\mathrm{Hdac} 4 w t$ mice was detected, demonstrating that the Hdac4bko mice were significantly hyperactive compared with littermate controls $\left({ }^{*} p<0.05\right.$; inset). To better delineate the alteration in locomotor activity, the data were analyzed in $5 \mathrm{~min}$ increments. Based on repeated measures with mixed-model analysis, there were significant group effects between Hdac4bko and Hdac $4 w t$ mice $\left(F_{(1,39)}=6.18\right.$; value of $\left.p=0.0173\right)$ and time effect $\left(F_{(23,897)}=109.44\right.$; value of $\left.p<0.0001\right)$ further demonstrating the hyperactivity of the Hdac4bko mice. C, Hdac4bko show a significant increase in time spent in the center of the maze as well as the open arms, and a significant decrease in time spent in the closed arms $\left({ }^{*} p<\right.$ 0.05; Hdac $4 w t, n=18$; Hdac4bko, $n=23$ ). D, Hdac4bko were less anxious than littermate Hdac4wt mice in the open-field test. The Hdac4bko mice spent significantly more time in the complete center and less time in the periphery compared with Hdac4wt mice ( $^{*} p<0.05$; Hdac $4 w t, n=18 ; H d a c 4 b k 0, n=23)$. $E$, The time spent freezing 90 min and $24 \mathrm{~h}$ after training was presented to examine STM and LTM, respectively, in the fear-conditioning paradigm. Results indicate a statistically significant decrease in LTM formation but not STM in the Hdac4bko compared with Hdac4wt mice ( ${ }^{*} p<0.05$; Hdac4wt, $n=8$; Hdac4bko, $n=13$ ). F, Hdac4bko mice are impaired in spatial learning in the Morris water maze. A probe trial was performed on day 11. Hdac4bko mice spent a similar amount of time in the four quadrants, while the Hdac $4 w t$ mice showed a preference for the target quadrant and spent significantly more time in the target quadrant $\left({ }^{*} p<0.05 ; H d a c 4 w t, n=11 ; H d a c 4 b k o, n=11\right) . G$, Hdac4bko mice show no differences from Hdac4wt mice in the average time to reach the platform during the $10 \mathrm{~d}$ training ( $\left.{ }^{*} p<0.05 ; H d a c 4 w t, n=11 ; H d a c 4 b k 0, n=11\right) . H$, Hdac4wt mice and Hdac4bko mice have similar swim speeds, and there were no significant differences in average swim velocity between the two groups $\left({ }^{*} p<0.05\right.$; Hdac4wt, $n=11$; Hdac4bko, $\left.n=11\right)$. For all experiments, error bars show SEM. field test, behavioral tasks that incorporate aspects of human anxiety and have predictive validity for anxiolytic drugs (Holmes, 2001; Crawley, 2008). In the elevated plus maze, the Hdac4bko mice spent significantly more time in the center and the open arms and significantly less time in the closed arms compared with Hdac4wt mice, suggesting a decrease in anxiety-like behavior (Fig. 2C). To further examine whether the loss of Hdac4 decreases anxiety-related behavior, we used the open-field test. In the open-field test, the Hdac4bko mice spent significantly more time in the complete center of the field and significantly less time in the periphery compared with Hdac4wt animals, consistent with a decrease in anxiety-related behavior (Fig. 2D).

Since previous data have suggested that HDAC inhibition augments learning and memory, we tested the Hdac4bko mice in context-dependent fear conditioning. In this behavioral test, mice learn to associate a context (the environment) with an aversive footshock. When animals are reexposed to the same environment (context) $24 \mathrm{~h}$ later, they generate a fear response in which they "freeze" (Phillips and LeDoux, 1992). Baseline freezing between Hdac4bko and Hdac4wt mice was indistinguishable (data not shown). Ninety minutes after the training session, we tested the animals for STM formation and found that Hdac4bko mice displayed indistinguishable freezing behavior to Hdac4wt mice (Fig. 2 E). In contrast, $24 \mathrm{~h}$ after the training when the Hdac4bko mice were examined for LTM, they showed significantly less freezing in response to the context compared with Hdac4wt mice, suggestive of a deficit in learning and memory (Fig. 2E). The decrease in freezing of the Hdac4bko mice was not due to differences in pain sensitivity to footshock between the Hdac4bko and Hdac4wt mice (data not shown). These data suggest that the behavioral impairments during fear conditioning observed with the Hdac4bko mice may be due to deficits in associative learning. To evaluate this possibility, we used the Morris water maze paradigm to test hippocampal-dependent learning and memory, independent of locomotor activity (Vorhees and Williams, 2006). During the training period (four trials per day for $10 \mathrm{~d}$ ), each animal was placed in the pool and allowed to swim ad libitum for up to $1 \mathrm{~min}$ or until the platform was located. The Hdac4bko mice were able to locate the platform as rapidly as Hdac4wt animals and showed no significant differences in swim velocity, indicating that the basic neurological functions needed for swimming and sight were normal (Fig. 2G,H). This was particularly important as previous work has shown that HDAC4 is essential for survival of retinal neurons (Chen and Cepko, 2009). A probe test was performed on day 11 in which the platform was removed and mice were allowed to swim ad libitum for 1 min to quantify the amount of time spent in each quadrant. We found that Hdac4wt mice spent significantly more time in the target quadrant where the submerged platform had been located, while in contrast the Hdac4bko mice did not prefer the target quadrant (Fig. $2 F$ ), suggesting that Hdac4bko mice have significant impairments in spatial learning and memory.

\section{Hdac $^{-/-}$KO mice display no overt behavioral phenotypes}

The constitutive $H d a c 5^{-1-}$ knock-out mice had been previously generated (Chang et al., 2004). We investigated whether the loss of Hdac5, another key member of the class II HDAC family, produced similar behavioral phenotypes as those observed in the HDAC4bko mice. We found that the $H d a c 5^{-1-}$ mice were indistinguishable from wild-type littermate controls, referred to as Hdac5wt, in motor coordination as assessed by the rotarod test (Fig. 3A). The Hdac5 ${ }^{-1-}$ and Hdac5wt mice displayed similar total locomotor activity for $120 \mathrm{~min}$ (Fig. 3B, inset), as well as 


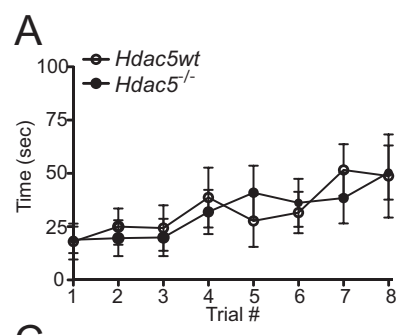

C


D
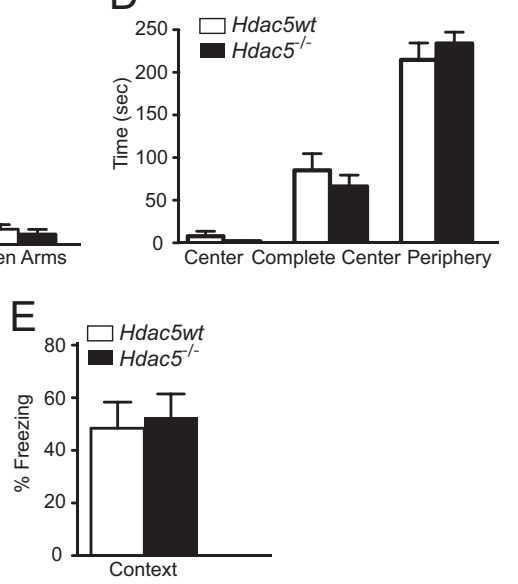

Figure 3. $H d a c 5^{-1-}$ mice have normal behavior including memory formation. $\boldsymbol{A}$, $H d a 5^{-1-}$ mice have normal motor coordination, staying on the rotarod as well as littermate $\mathrm{Hdac} 5 \mathrm{wt}$. The $\mathrm{Hdac} 5^{-1}$ mice were indistinguishable from Hdac5 wt mice in motor coordination as assessed by the rotarod test, as repeated measures with mixed-model analysis revealed no significant group effect $\left(F_{(1,6)}=0.06\right.$; value of $\left.p=0.8109\right)$. $\boldsymbol{B}$, The $\mathrm{Hdac5}-1$ - mice were examined for alterations in total locomotor activity for $120 \mathrm{~min}$, and using Student's $t$ test analysis, there was no significant difference between $\mathrm{Hdac} 5^{-1-}$ and $\mathrm{Hdac} 5 \mathrm{Wt}$ mice. We also assessed ambulation in 5 min increments and, based on repeated measures with mixed-model analysis, found no significant group effect between $\mathrm{Hdac5}-1-$ and $\mathrm{Hdac5wt}$ mice $\left(F_{(1,6)}=\right.$ 5.87 ; value of $p=0.0517)$, while there is a significant time effect $\left(F_{(23,138)}=38.20\right.$; value of $p<0.0001)$. $C$, In the elevated plus maze test, $\mathrm{Hdac}^{-1}$ - mice show no significant difference in time spent in the center of the maze, closed arms, or open arms compared with Hdac5wt mice. $\boldsymbol{D}$, In the open-field test, the $H d a c 5^{-I}$ mice spent a similar amount of time in the complete center and periphery compared with $\mathrm{Hdac} 5 \mathrm{wt}$ mice. $\mathrm{E}, \mathrm{HdaC5^{-1- }}$ mice show normal context-dependent fear conditioning. The time spent freezing $24 \mathrm{~h}$ after training in a one-trial fear-conditioning paradigm was assessed. Results indicate no difference in fear memory in the $\mathrm{HdaC5}^{-1-}$ mice compared with Hdac5wt mice $\left(\mathrm{Hdac5}{ }^{-1-}, n=7 ; \mathrm{Hdac5wt}, n=8\right)$.

when data was analyzed in $5 \mathrm{~min}$ increments (Fig. 3B). The $H$ dac $5^{-1-}$ mice also exhibited normal anxiety-related behaviors as assessed in the elevated plus maze (Fig. 3C) and open-field test (Fig. 3D) relative to Hdac5wt mice. To examine the involvement of HDAC5 in learning and memory, we tested the mice in the fear conditioning behavioral task. We found that the Hdac5 ${ }^{-1-}$ had similar freezing behavior in context-dependent fear conditioning to Hdac5wt mice (Fig. 3E). In addition, the Hdac5 ${ }^{-1-}$ had indistinguishable levels of baseline freezing and pain sensitivity to footshock compared with Hdac5wt mice (data not shown). These data show that, in striking contrast to the loss of Hdac4, loss of Hdac5 does not impact context-dependent fear conditioning, underscoring the specificity of our observations.

\section{Impaired long-term synaptic plasticity in Hdac4bko mice}

Long-term changes in synaptic plasticity are widely believed to be the cellular basis for learning and memory. Therefore, we examined whether the impaired learning and memory behaviors seen after the loss of Hdac4 were associated with changes in LTP by performing field recordings on hippocampal slices prepared from Hdac4bko and Hdac4wt mice. LTP was induced in the CA1


Figure 4. Impaired hippocampal plasticity in Hdac4bko mice. A, Hdac4bko mice display impaired LTP at Schaffer collateral/CA1 pyramidal cell synapses compared with Hdac4wt animals as assessed by a two-way ANOVA with repeated measures $\left({ }^{*} p<0.05\right.$; Hdac $4 w t, n=7$; Hdac4bko, $n=8$ ). $\boldsymbol{B}$, The averaged fEPSP plotted against presynaptic volley amplitude shows no significant difference in input- output function of CA1-fEPSPs in Hdac4bko mice compared with Hdac4wt controls (Hdac4wt, $n=7$; Hdac $4 b k 0, n=8$ ). Input-output slopes were fit by linear regression and between group slopes were subsequently compared using unpaired $t$ tests. C, The averaged paired-pulse facilitation for Hdac4wt and Hdac4bko slices at various intervals at SC-CA1 synapses. Using Student's $t$ test, significant decreases in Hdac4bko mice were observed at the first three interstimulus interval data $(20,30$, and $50 \mathrm{~ms})\left({ }^{*} p<0.05\right.$; Hdac $4 w t, n=7 ;$ Hdac4bko, $n=8$ ).

of the hippocampus by three trains of theta burst stimulation on the Schaffer collateral pathway. The Hdac4bko and Hdac4wt mice developed an immediate increase in the field EPSP (fEPSP) slope $\left({ }^{\star} p<0.05\right)$, although the magnitude of enhancement of LTP was markedly reduced in Hdac4bko mice, suggesting a role for HDAC4 in the modulation of long-term synaptic plasticity in the hippocampus (Fig. 4A). We investigated whether the decreased LTP observed in Hdac4bko mice might be associated with alterations in basal synaptic properties. Input-output curves, generated by plotting fEPSP slopes against fiber volley amplitudes, were unchanged in Hdac4bko mice compared with Hdac4wt mice, indicating that basal synaptic transmission is largely unaltered in Hdac4bko mice (Fig. 4B). However, PPF, a short-term form of synaptic plasticity associated with the probability of neurotransmitter release, was decreased in Hdac4bko mice over a range of interevent intervals $(20,30$, and $50 \mathrm{~ms})$ consistent with an increase in presynaptic release probability, suggesting that loss of Hdac4 leads to alterations in presynaptic function (Fig. 4C). Together, these results indicate that the deficit seen in LTP after loss of Hdac4 is presumably independent of the increase in release probability as we could not detect a corresponding increase in input-output curves.

\section{Loss of Hdac4 or Hdac5 does not impact basal synaptic transmission}

To further evaluate whether the LTP impairments observed with the Hdac4bko mice may be linked to differences in basal synaptic properties, we investigated whether loss of Hdac4 impacts unitary synaptic properties using whole-cell voltage-clamp recordings in primary hippocampal cultures. Knockdown of Hdac4 expression was achieved by infecting homozygous Hdac $4^{\text {loxP/loxP }}$ hippocampal cultures with a lentivirus expressing Cre recombinase at 4 
DIV and then recording on 14-17 DIV. The successful knockdown of Hdac4 in culture following lentiviral-Cre expression was confirmed by quantitative PCR (data not shown). The knockdown of Hdac4 did not impact either the frequencies or amplitudes of mEPSCs, indicating lack of alterations in parameters such as excitatory synapse numbers, spontaneous release probability, as well as postsynaptic AMPA receptor numbers (Fig. $5 A-C$ ). We also investigated whether loss of Hdac5 impacts unitary synaptic properties in primary hippocampal cultures. We did not detect any alterations in mEPSC properties of Hdac5 ${ }^{-1-}$ compared with Hdac5wt hippocampal neurons (Fig. 5D-F). Together, these results support the premise that HDAC4 or HDAC5 are not required for maintenance of unitary properties of synaptic transmission in the hippocampus.

\section{Discussion}

Our results provide the first genetic evidence that an individual class IIa HDAC, namely HDAC4, is specifically required for learning and memory and synaptic plasticity in mice. Although these findings appear to contrast with previous work showing that HDAC inhibition is a negative regulator of these processes (Korzus et al., 2004; Fischer et al., 2007; Guan et al., 2009), HDAC inhibition has largely been achieved with sodium butyrate, valproate, and suberoylanilide hydroxamic acid (SAHA), agents that preferentially target class I, but not class IIa or IIb, HDACs (Kilgore et al., 2010). Furthermore, our results demonstrate unique and functionally non-redundant roles for HDAC4 and HDAC5 in learning and memory as well as synaptic plasticity, suggesting that broad-scale manipulation of class I or class II HDACs may have differing effects on these processes.

The positive role for HDAC4 in hippocampal-dependent learning and memory, as well as well as LTP, agrees with recent work linking a haploinsufficiency of HDAC4 with brachydactyly mental retardation syndrome (Williams et al., 2010). Our findings imply that inhibition of HDAC4 activity may exert detrimental effects on learning and memory, as well related synaptic processes. Our initial assumption was that HDAC4 involvement in transcriptional control mediated the learning and memory and synaptic plasticity deficits. However, the use of microarray analysis to identify genes differentially regulated in the hippocampus by the loss of Hdac4 did not yield significant changes in gene expression (data not shown). Moreover, our findings that LTP is impaired immediately after its induction, at a time point typically insensitive to transcriptional inhibitors, in the Hdac4bko mice further suggests that these deficits are not due to acute activitydependent regulation of transcriptional processes. The lack of gene expression changes by our microarray analysis suggests that the loss of Hdac4 is not altering the expression of a signaling molecule that inhibits early-phase LTP but rather that HDAC4 may play a role in nontranscriptional processes involved in LTP. Future studies will be necessary to better delineate the involvement of HDAC4 in these processes to determine whether HDAC4's activity-dependent shuttling, cytoplasmic functioning, or possibly deacetylation of endogenous proteins other than histones are involved.

Depending on the phosphorylation state and cellular location, class IIa HDACs associate with myocyte enhancer factor 2 (MEF2) proteins or the chaperone protein 14-3-3 (Haberland et al., 2009). Previously, we analyzed the role of MEF2C in neurons by generating a brain-specific deletion of MEF2C and showed that $\mathrm{MEF} 2 \mathrm{C}$ plays a role in hippocampal-dependent learning and memory by suppressing the number of excitatory synapses and thus regulating basal and evoked synaptic transmission (Barbosa et al., 2008). Therefore, in future work, it will be critical to address whether HDAC4-dependent regulation of learning and memory requires $\mathrm{MEF} 2 \mathrm{C}$ function.

Behavioral analysis of brain-specific Hdac4bko mice also revealed impairments in motor coordination, hyperactivity, and decreased anxiety-like behaviors. HDAC4 has been associated with muscle and bone development (Miska et al., 1999, 2001; Lu et al., 2000; McKinsey et al., 2000; Vega et al., 2004; Arnold et al., 2007) through specific effects mediated by its deactylase domain (Rajan et al., 2009). The loss of Hdac4 selectively in the brain resulted in decreased performance on the rotarod test, suggesting impairments in motor coordination. Together, these findings highlight the critical role of HDAC4 in neuromuscular development and function. In contrast, the finding that loss of Hdac4 in mice results in a decrease in anxiety-related behavior reveals an unexpected association between HDAC inhibition and anxiety. Chronic infusion of SAHA, a class I HDAC inhibitor, into the basolateral amygdala of adult mice has been shown to heighten anxiety-related behavior (Adachi et al., 2009). However, it has also been suggested that a putative link between HDACs and anxiety may encompass processes related to early life events. Early life experiences can impact adult behavior and have been suggested to involve epigenetic mechanisms. Administration of 
trichostatin A (TSA), a broad-acting HDAC inhibitor, into adult rodents reverses anxiety-related behavioral alterations that were the result of deficient maternal care (Weaver et al., 2004, 2006). These data support the hypothesis that the beneficial effects of trichostatin A on anxiety-related behavior may be due to the selective reduction of HDAC4. The ability of HDAC4 to negatively regulate anxiety-related behavior, as well as the demonstration it does not adversely impact synaptic transmission, may make it an attractive potential target for anxiety disorders.

We found that the global loss of Hdac5 does not impact any of the examined behavioral measures, including learning and memory. HDAC5 has been previously implicated in reward-related behaviors in that it is downregulated by chronic cocaine or stress, and loss of Hdac5 in mice results in hypersensitivity to chronic cocaine and stress (Renthal et al., 2007). Together, these findings suggest that HDAC5 does not play a significant role in hippocampaldependent and other associated behaviors under normal conditions but may be a crucial factor under certain disease states such as addiction or chronic stress.

Whole-cell voltage-clamp recordings from hippocampal neurons showed that loss of Hdac4 or Hdac5 does not impact unitary synaptic transmission. Recently, our group has demonstrated that the pan HDAC inhibitor drug, TSA, triggers a decrease in mEPSC frequency in mature hippocampal neurons without altering synapse numbers, via a mechanism dependent on new gene expression (Nelson et al., 2006). A subsequent study showed that deletion of $H d a c 2$, but not Hdac1, in mature hippocampal neurons results in a decrease in the frequency of mEPSCs with no change in synapse number, similar to the deficits in synaptic transmission observed with TSA (Akhtar et al., 2009). In these studies, the lack of an effect of HDAC1 on synaptic transmission in mature hippocampal cultures may not be that surprising given the low level of Hdacl expression in postmitotic hippocampal neurons. In contrast, Hdac4 and Hdac5 are expressed at high levels in postmitotic hippocampal neurons similar to expression of Hdac2 (Broide et al., 2007). Yet the loss of Hdac4 or Hdac5 did not impact unitary synaptic transmission, suggesting specificity for individual HDACs in these processes. Together with this earlier work, our findings suggest that class I HDACs that are expressed in postmitotic neurons play critical roles in regulating unitary properties of excitatory neurotransmission as well as plasticity, while class II HDACs, which shuttle between the nucleus and cytoplasm in an activity-dependent manner, are not involved in unitary properties of synaptic function. Future work examining other individual HDACs in mature neurons will be critical to test the premise that class I and II HDACs are specialized with respect to their roles in basal neurotransmission.

HDAC inhibition has been linked to beneficial effects in learning and memory as well as synaptic plasticity for a range of neurological and psychiatric disorders. Our finding that HDAC4 acts as a positive regulator of learning and memory as well as synaptic plasticity highlights the complex roles HDACs play in the CNS and underscores the critical need for understanding the role of individual members of this gene family in the brain. Moreover, since transport of HDAC4 from the nucleus to the cytoplasm is regulated by calcium signaling (Backs et al., 2008), and alterations in calcium-dependent pathways have been linked to a wide variety of brain disorders with learning and memory deficits (Bezprozvanny and Hayden, 2004), it will be interesting to examine HDAC4 expression and activity in these pathological conditions. Given the recent efforts to develop HDAC inhibitors for a variety of disorders, the data from the Hdac4 and Hdac5 mutant mice reveal specific roles in neuronal plasticity deviating from the role of class I HDACs in these processes and emphasize the need for caution inferred by broad-scale pharmacological inhibition.

\section{References}

Abel T, Zukin RS (2008) Epigenetic targets of HDAC inhibition in neurodegenerative and psychiatric disorders. Curr Opin Pharmacol 8:57-64.

Adachi M, Autry AE, Covington HE 3rd, Monteggia LM (2009) MeCP2mediated transcription repression in the basolateral amygdala may underlie heightened anxiety in a mouse model of Rett syndrome. J Neurosci 29:4218-4227.

Akhtar MW, Raingo J, Nelson ED, Montgomery RL, Olson EN, Kavalali ET, Monteggia LM (2009) Histone deacetylases 1 and 2 form a developmental switch that controls excitatory synapse maturation and function. J Neurosci 29:8288-8297.

Alarcón JM, Malleret G, Touzani K, Vronskaya S, Ishii S, Kandel ER, Barco A (2004) Chromatin acetylation, memory, and LTP are impaired in $\mathrm{CBP}^{+/-}$mice: a model for the cognitive deficit in Rubinstein-Taybi syndrome and its amelioration. Neuron 42:947-959.

Arnold MA, Kim Y, Czubryt MP, Phan D, McAnally J, Qi X, Shelton JM, Richardson JA, Bassel-Duby R, Olson EN (2007) MEF2C transcription factor controls chondrocyte hypertrophy and bone development. Dev Cell 12:377-389.

Backs J, Backs T, Bezprozvannaya S, McKinsey TA, Olson EN (2008) Histone deacetylase 5 acquires calcium/calmodulin-dependent kinase II responsiveness by oligomerization with histone deacetylase 4 . Mol Cell Biol 28:3437-3445.

Barbosa AC, Kim MS, Ertunc M, Adachi M, Nelson ED, McAnally J, Richardson JA, Kavalali ET, Monteggia LM, Bassel-Duby R, Olson EN (2008) MEF2C, a transcription factor that facilitates learning and memory by negative regulation of synapse numbers and function. Proc Natl Acad Sci U S A 105:9391-9396.

Bezprozvanny I, Hayden MR (2004) Deranged neuronal calcium signaling and Huntington disease. Biochem Biophys Res Commun 322:1310-1317.

Borrelli E, Nestler EJ, Allis CD, Sassone-Corsi P (2008) Decoding the epigenetic language of neuronal plasticity. Neuron 60:961-974.

Broide RS, Redwine JM, Aftahi N, Young W, Bloom FE, Winrow CJ (2007) Distribution of histone deacetylases $1-11$ in the rat brain. J Mol Neurosci 31:47-58.

Chang S, McKinsey TA, Zhang CL, Richardson JA, Hill JA, Olson EN (2004) Histone deacetylases 5 and 9 govern responsiveness of the heart to a subset of stress signals and play redundant roles in heart development. Mol Cell Biol 24:8467-8476.

Chen B, Cepko CL (2009) HDAC4 regulates neuronal survival in normal and diseased retinas. Science 323:256-259.

Crawley JN (2008) Behavioral phenotyping strategies for mutant mice. Neuron 57:809-818.

Dittgen T, Nimmerjahn A, Komai S, Licznerski P, Waters J, Margrie TW, Helmchen F, Denk W, Brecht M, Osten P (2004) Lentivirus-based genetic manipulations of cortical neurons and their optical and electrophysiological monitoring in vivo. Proc Natl Acad Sci U S A 101:18206-18211.

Fischer A, Sananbenesi F, Wang X, Dobbin M, Tsai LH (2007) Recovery of learning and memory is associated with chromatin remodelling. Nature 447:178-182.

Gemelli T, Berton O, Nelson ED, Perrotti LI, Jaenisch R, Monteggia LM (2006) Postnatal loss of methyl-CpG binding protein 2 in the forebrain is sufficient to mediate behavioral aspects of Rett syndrome in mice. Biol Psychiatry 59:468-476.

Guan JS, Haggarty SJ, Giacometti E, Dannenberg JH, Joseph N, Gao J, Nieland TJ, Zhou Y, Wang X, Mazitschek R, Bradner JE, DePinho RA, Jaenisch R, Tsai LH (2009) HDAC2 negatively regulates memory formation and synaptic plasticity. Nature 459:55-60.

Haberland M, Montgomery RL, Olson EN (2009) The many roles of histone deacetylases in development and physiology: implications for disease and therapy. Nat Rev Genet 10:32-42.

Holmes A (2001) Targeted gene mutation approaches to the study of anxiety-like behavior in mice. Neurosci Biobehav Rev 25:261-273.

Jiang Y, Langley B, Lubin FD, Renthal W, Wood MA, Yasui DH, Kumar A, Nestler EJ, Akbarian S, Beckel-Mitchener AC (2008) Epigenetics in the nervous system. J Neurosci 28:11753-11759.

Kavalali ET, Klingauf J, Tsien RW (1999) Activity-dependent regulation of synaptic clustering in a hippocampal culture system. Proc Natl Acad Sci U S A 96:12893-12900. 
Kilgore M, Miller CA, Fass DM, Hennig KM, Haggarty SJ, Sweatt JD, Rumbaugh G (2010) Inhibitors of class 1 histone deacetylases reverse contextual memory deficits in a mouse model of Alzheimer's disease. Neuropsychopharmacology 35:870-880.

Korzus E, Rosenfeld MG, Mayford M (2004) CBP histone acetyltransferase activity is a critical component of memory consolidation. Neuron 42:961-972.

Kumar A, Choi KH, Renthal W, Tsankova NM, Theobald DE, Truong HT, Russo SJ, Laplant Q, Sasaki TS, Whistler KN, Neve RL, Self DW, Nestler EJ (2005) Chromatin remodeling is a key mechanism underlying cocaineinduced plasticity in striatum. Neuron 48:303-314.

Levenson JM, O’Riordan KJ, Brown KD, Trinh MA, Molfese DL, Sweatt JD (2004) Regulation of histone acetylation during memory formation in the hippocampus. J Biol Chem 279:40545-40559.

Livak KJ, Schmittgen TD (2001) Analysis of relative gene expression data using real-time quantitative PCR and the $2^{-\Delta \Delta C(\mathrm{~T})}$ method. Methods 25:402-408.

Lu J, McKinsey TA, Zhang CL, Olson EN (2000) Regulation of skeletal myogenesis by association of the MEF2 transcription factor with class II histone deacetylases. Mol Cell 6:233-244.

Luikart BW, Nef S, Virmani T, Lush ME, Liu Y, Kavalali ET, Parada LF (2005) TrkB has a cell-autonomous role in the establishment of hippocampal Schaffer collateral synapses. J Neurosci 25:3774-3786.

McKinsey TA, Zhang CL, Lu J, Olson EN (2000) Signal-dependent nuclear export of a histone deacetylase regulates muscle differentiation. Nature 408:106-111.

Miska EA, Karlsson C, Langley E, Nielsen SJ, Pines J, Kouzarides T (1999) HDAC4 deacetylase associates with and represses the MEF2 transcription factor. EMBO J 18:5099-5107.

Miska EA, Langley E, Wolf D, Karlsson C, Pines J, Kouzarides T (2001) Differential localization of HDAC4 orchestrates muscle differentiation. Nucleic Acids Res 29:3439-3447.

Monteggia LM, Barrot M, Powell CM, Berton O, Galanis V, Gemelli T, Meuth S, Nagy A, Greene RW, Nestler EJ (2004) Essential role of brain-derived neurotrophic factor in adult hippocampal function. Proc Natl Acad Sci U S A 101:10827-10832.

Nelson ED, Kavalali ET, Monteggia LM (2006) MeCP2-dependent transcriptional repression regulates excitatory neurotransmission. Curr Biol $16: 710-716$

Phillips RG, LeDoux JE (1992) Differential contribution of amygdala and hippocampus to cued and contextual fear conditioning. Behav Neurosci 106:274-285.

Potthoff MJ, Wu H, Arnold MA, Shelton JM, Backs J, McAnally J, Richardson JA, Bassel-Duby R, Olson EN (2007) Histone deacetylase degradation and MEF2 activation promote the formation of slow-twitch myofibers. J Clin Invest 117:2459-2467.

Rajan I, Savelieva KV, Ye GL, Wang CY, Malbari MM, Friddle C, Lanthorn TH, Zhang W (2009) Loss of the putative catalytic domain of HDAC4 leads to reduced thermal nociception and seizures while allowing normal bone development. PLoS One 4:e6612.

Renthal W, Maze I, Krishnan V, Covington HE 3rd, Xiao G, Kumar A, Russo SJ, Graham A, Tsankova N, Kippin TE, Kerstetter KA, Neve RL, Haggarty SJ, McKinsey TA, Bassel-Duby R, Olson EN, Nestler EJ (2007) Histone deacetylase 5 epigenetically controls behavioral adaptations to chronic emotional stimuli. Neuron 56:517-529.

Shelton JM, Lee MH, Richardson JA, Patel SB (2000) Microsomal triglyceride transfer protein expression during mouse development. J Lipid Res 41:532-537.

Vecsey CG, Hawk JD, Lattal KM, Stein JM, Fabian SA, Attner MA, Cabrera SM, McDonough CB, Brindle PK, Abel T, Wood MA (2007) Histone deacetylase inhibitors enhance memory and synaptic plasticity via CREB: CBP-dependent transcriptional activation. J Neurosci 27:6128-6140.

Vega RB, Matsuda K, Oh J, Barbosa AC, Yang X, Meadows E, McAnally J, Pomajzl C, Shelton JM, Richardson JA, Karsenty G, Olson EN (2004) Histone deacetylase 4 controls chondrocyte hypertrophy during skeletogenesis. Cell 119:555-566.

Vorhees CV, Williams MT (2006) Morris water maze: procedures for assessing spatial and related forms of learning and memory. Nat Protoc $1: 848-858$.

Weaver IC, Cervoni N, Champagne FA, D’Alessio AC, Sharma S, Seckl JR, Dymov S, Szyf M, Meaney MJ (2004) Epigenetic programming by maternal behavior. Nat Neurosci 7:847-854.

Weaver IC, Meaney MJ, Szyf M (2006) Maternal care effects on the hippocampal transcriptome and anxiety-mediated behaviors in the offspring that are reversible in adulthood. Proc Natl Acad Sci U S A 103:3480-3485.

Williams SR, Aldred MA, Der Kaloustian VM, Halal F, Gowans G, McLeod DR, Zondag S, Toriello HV, Magenis RE, Elsea SH (2010) Haploinsufficiency of HDAC4 causes brachydactyly mental retardation syndrome, with brachydactyly type E, developmental delays, and behavioral problems. Am J Hum Genet 87:219-228. 